\title{
Zu den Feindbildern auf den Zauberstäben des Mittleren Reiches und der Zweiten Zwischenzeit
}

\author{
HARTWig AltenMüller
}

\begin{abstract}
The ivory wands of the Middle Kingdom display various creatures to be annihilated by protective deities, especially snakes and human foes. It seems that the snakes pictured on the ivory wands represent chaotic numina of primeval times which endanger the uncreated and the animate world. The human foes on the other side are foreigners like Libyans, Asians, Nubians and rebels. It is interesting to note that the figures of the human foes are modelled after an archetype which is taken from the royal monuments showing the king smiting his enemies. While the foes represented on the royal monuments are put down by the king in the shape of a divine bull, lion, sphinx or griffin, the enemies of the ivory wands are smitten by divine forces, among them the hippopotamus-goddess, the lion, and a cat-goddess. The investigation concerns the religious background of the massacre, which is done by the divine forces on snakes and human foes. It is proposed to identify the fighting animals as protective deities originating in part from stellar constellations or decans protecting the mother and her child at the beginning of lifetime.
\end{abstract}

Keywords: Middle Kingdom Egypt, Second Intermediate period, apotropaic wand, snake, foe, hippopotamus, lion, cat, decans, constellations, magic

English title: A Note on Foes as Represented on the Apotropaic Ivory Wands of the Middle Kingdom and the Second Intermediate Period

Hartwig Altenmüller, Archäologisches Institut der Universität Hamburg, Arbeitsbereich Ägyptologie, Hamburg; altenmueller@uni-hamburg.de

Die sog. Zauberstäbe des Mittleren Reiches und der zweiten Zwischenzeit, die Mutter und Kind bei Tag und bei Nacht vor Schlangen und anderen gefährlichen Wesen schützen, geben der Forschung zahlreiche Fragen auf. Selbst ihre Bezeichnung bereitet noch Schwierigkeiten. Die meist aus dem unteren Eckzahn des Nilpferds gewonnenen Zaubergeräte werden „Zauberstab“"

\footnotetext{
${ }^{1} R \ddot{A} R G: 880$.
} 
„Zaubermesser“2 , „Apotropaion“33 oder „magischer Nilpferdstoßzahn““4 genannt ${ }^{5}$. Hier soll der Begriff „gebogener Zauberstab“ verwendet werden.

Die gebogenen Zauberstäbe bestehen vorwiegend aus dem flach geschliffenen gebogenen Nilpferdstoßzahn und sind für die Verwendung als Zauberstab mit eingeritzten Figuren versehen. Am spitzen Ende des Nilpferdstoßzahns befindet sich ein üblicherweise geritzter, gelegentlich auch plastisch ausgearbeiteter Fuchskopf (Kairo JE 91413). Das stumpfe Ende ist in der Regel mit einem Pantherkopf versehen, der ebenfalls plastisch ausgearbeitet sein kann. Die Ober- und Unterseite des flach geschliffenen Nilpferdstoßzahns bilden das Dekorationsfeld mit Götterfiguren (Kairo CG 9438). Die in das Elfenbein eingeritzten Götterfiguren sind fast immer auf der inneren Linie des konvexen Bogens des Nilpferdstoßzahns angebracht und zu Götterprozessionen geordnet, die sich in einem nach oben geführten Bogen bewegen. Nur in seltenen Fällen ist der Zauberstab nach unten gebogen, so dass die Götterprozessionen auf der äußeren konkaven Biegung zunächst ab- und dann ansteigend marschieren (vgl. Abb. 1a und London BM 18175).

Die abgebildeten Gottheiten weisen zum überwiegenden Teil Tiergestalt auf. Nur wenige Figuren sind anthropomorph gebildet, besitzen dann aber meist Tierköpfe als Hinweis auf ihre Abstammung von einer ursprünglichen Tiergottheit. Die Tiermächte, darunter auch Fabelwesen, führen Messer oder zerfleischen Schlangen mit ihren Mäulern. Einige werden beim Niederschlagen, Niedertreten und Töten von menschlichen Feinden gezeigt.

Die magische Bedeutung der gebogenen Zauberstäbe aus Elfenbein ergibt sich aus den Beischriften, die meist auf der Rückseite eingeritzt sind und den individuellen Schutz für eine bestimmte Person formulieren. Die übliche Formel lautet ${ }^{6}$ : Rezitation seitens der Schutzgötter: Wir sind gekommen und breiten den Schutz des Lebens über dem Kind X, geboren von der Herrin des Hauses NN aus, oder: Rezitation seitens der Schutzgötter: Wir sind gekommen und breiten den Schutz des Lebens über der Herrin des Hauses X, geboren von NN aus. Eine nicht der Norm entsprechende Beischrift auf dem Zauberstab Kopenhagen NM 7795 (vgl. Tab. 1) lautet einmal so: Rezitation seitens der (Mächte, die) den Kopf des Feindes/der Feindin abschneiden, die das Zimmer der Kinder der NN betreten ${ }^{7}$. Wie die Beischriften besagen, besteht der eigentliche Zweck der Zauberstäbe darin, Mutter und Kind bei Tag und bei Nacht zu beschützen.

Über den performativen Gebrauch der Zauberstäbe informieren mehrere Quellen. Besonders instruktiv ist im Amduat des Neuen Reiches das Bild eines Gefolgsmanns des Sonnengottes, der als „Stundendurchfahrer“ ( $\left.d_{3 j} j w w t\right)$ mit einem Zauberstab in der Hand an der Nachtfahrt des Sonnengottes teilnimmt ${ }^{8}$. In der vierten Stunde der Nacht wird

2 Bissing 1934: 196 (Bd. I).

${ }^{3}$ Altenmüller 1965: 8 (Bd. I).

${ }^{4}$ Gnirs 2009: 128.

${ }^{5}$ Im angelsächsischen Raum werden die Begriffe „,ivory wand“ (Legge 1905; 1906), ,apotropaic wand“ (Pinch 1994: 78), ,birth hippopotamus-tusks“ (Quirke 2005: 100) gebraucht.

${ }^{6} \mathrm{Zu}$ den Formeln: Altenmüller 1965: 64-70 (Bd. I); vgl. Altenmüller 2015: 22.

${ }^{7}$ Altenmüller 1965: 69 (Bd. I); 46 (Bd. II).

${ }^{8}$ Hornung 1963: 11 (Bd. I); 24 (Bd. II), Nr. 63. 
Tab. 1. Liste der im Text behandelten Zauberstäbe

\begin{tabular}{|c|c|c|}
\hline Museum Nummer & Länge & Literaturverzeichnis \\
\hline Baltimore WAM 71510 & $37 \mathrm{~cm}$ & Altenmüller 1965: 3 (Bd. II) \\
\hline Basel BSAe 991 & c. $55 \mathrm{~cm}$ & Altenmüller 1986: 1-27 \\
\hline Berlin ÄM 14207 & $41 \mathrm{~cm}$ & Altenmüller 1965: 11-12 (Bd. II) \\
\hline Jerusalem IAA 1948-1391 & $25 \mathrm{~cm}$ & Altenmüller 1965: 70-71 (Bd. II) \\
\hline Kairo CG 9435 & $34 \mathrm{~cm}$ & Altenmüller 1965: 38 (Bd. II) \\
\hline Kairo CG 9438 & c. $20 \mathrm{~cm}$ & Altenmüller 1965: 40-41 (Bd. II) \\
\hline Kairo JE 68773 & $16,8 \mathrm{~cm}$ & Altenmüller 1983: 40 (Bd. II) \\
\hline Kairo JE 91413 & c. $40 \mathrm{~cm}$ & Arnold, Settgast 1966: 86, Taf. 22 \\
\hline Kairo E. 2007.04.58 & $26 \mathrm{~cm}$ & Polz et al. 1999: 391, Abb. 18 \\
\hline Kofler A 101 & $17,3 \mathrm{~cm}$ & Altenmüller 1965: 44-45 (Bd. II) \\
\hline Kopenhagen NM 7795 & $28 \mathrm{~cm}$ & Altenmüller 1965: 45-46 (Bd. II) \\
\hline London BM 18175 & $46 \mathrm{~cm}$ & Altenmüller 1965: 50-52 (Bd. II) \\
\hline London UC 16384 & $11 \mathrm{~cm}$ & Altenmüller 1965: 65 (Bd. II) \\
\hline London UC 15917 & $20 \mathrm{~cm}$ & Altenmüller 1965: 66-67 (Bd. II) \\
\hline Louvre E 3614 + MMA 26.7.1288 a-b & $48 \mathrm{~cm}$ & Altenmüller 1965: 102-103 (Bd. II) \\
\hline Louvre E 20611 & $29,3 \mathrm{~cm}$ & Vandier 1969: 43-44, Abb. 3 \\
\hline Manchester 1799 & c. $20 \mathrm{~cm}$ & Altenmüller 1965: 110 (Bd. II) \\
\hline MMA 15.3.197 & $26,8 \mathrm{~cm}$ & Altenmüller 1965: 78-79 (Bd. II) \\
\hline MMA 15.3.951A & $6,6 \mathrm{~cm}$ & Altenmüller 1965: 81 (Bd. II) \\
\hline MMA 30.8.218 & $34 \mathrm{~cm}$ & Altenmüller 1965: 89-90 (Bd. II) \\
\hline MMA 32.8 .4 & $8 \mathrm{~cm}$ & Altenmüller 1965: 92-93 (Bd. II) \\
\hline München ÄS 2952 & $37 \mathrm{~cm}$ & Altenmüller 1965: 74-75 (Bd. II) \\
\hline
\end{tabular}

der Zauberstab einmal in Betrieb genommen. Die dort „Hetepu“ genannte Gottheit setzt einen Zauberstab ein, um den Sonnengott im Schlangenreich von Rosetau vor den dort hausenden Schlangen zu beschützen?.

Mit dem Gebrauch der Zauberstäbe in der Nacht lässt sich dann auch erklären, dass in einem Gerätefries des Mittleren Reiches ein Zauberstab neben anderen Gerätschaften eines Schlafzimmers auf einem Bett abgelegt ist ${ }^{10}$ und dass im Grab des Rechmire aus der frühen 18. Dynastie drei gebogene Zauberstäbe unter dem Bett einer Schlafzimmereinrichtung liegen ${ }^{11}$.

\footnotetext{
${ }^{9}$ Hornung 1963: 72 (Bd. I); 88 (Bd. II), Nr. 312.

${ }^{10}$ Boston MFA 20.1823: Terrace 1967: Taf. 15.

${ }^{11}$ Davies 1943: Taf. 37 (Bd. II).
} 
Der gebogene Zauberstab in der Hand einer Amme im Grab des Thothotep von el Berscheh verdeutlicht, dass das Gerät zur Grundausstattung von Ammen gehört ${ }^{12}$. Die Grabbilder des Bebi aus Elkab zeigen die Handhabung der Zauberstäbe genauer. Ammen $\left(m n^{\top} t\right)$ und Wärterinnen (hnmt) halten Zauberstäbe in die Höhe und schützen - gewiss unter der Rezitation von Zaubertexten - den Grabherrn und seine Frau sowie die Kinder der Familie ${ }^{13}$.

Eine große Unsicherheit besteht in der Frage, wer die Gottheiten im Bildprogramm der Zauberstäbe sind und wie sie heißen und welche Feinde von den Gottheiten vernichtet werden. Bisher unbekannt sind auch die Zaubertexte, die zur Handlung mit den Zauberstäben rezitiert wurden. Es wird sogar vermutet, dass der Zauber mit den Zauberstäben ganz ohne schriftlich fixierte Texte auskam ${ }^{14}$. Im Folgenden soll der Versuch unternommen werden, die Rolle der Schutzgottheiten bei der Bekämpfung der Feinde zu bestimmen und diese nach Möglichkeit zu identifizieren.

\section{DIE FEINDE IN TIERGESTALT}

Die größten Feinde, gegen die sich die Gottheiten der magischen Zauberstäbe wenden, sind die Schlangen, deren Bild das Bildprogramm der Zauberstäbe beherrscht ${ }^{15}$. Bei den Schlangen handelt es sich um Wesen der Urzeit, die die Schöpfung und die Welt bedrohen und vor denen man sich fürchtet, die aber auch hilfreich sein können. Nicht immer ist klar zu unterscheiden, welche Schlangen als Feinde und welche als Schutzgottheiten aufzufassen sind. Zu den feindlichen Schlangen zählen hauptsächlich jene Schlangen, die aus langgezogenen und sich dahin schlängelnden Linien von Schlangenleibern bestehen. Sie werden von den Mächten der Zauberstäbe in die Flucht gejagt oder vernichtet. Die mächtigen Wesen gehen mit dem Messer gegen die Schlangen vor, zerbeißen sie mit ihren Zähnen und verschlingen sie (vgl. Abb. 1b). Nur selten wehren sich die Schlangen gegen ihre Angreifer. Meist sind sie überwunden und wehrlos, werden gepackt, zerquetscht und gefressen. Größere Schlangen, deren Leib detailliert wiedergegeben ist, sind vermutlich in den meisten Fällen Schutzgottheiten. Doch lässt sich eine letzte Sicherheit nicht gewinnen, da entsprechende Texte fehlen.

Ein kurzer Zauberstab aus Edfu aus Lehm, der durch die Verwendung von leicht formbaren Material seine Zugehörigkeit zum täglichen Leben überzeugend nachweist und daher ad hoc hergestellt worden sein kann, enthält das Bild einer von Messern zerstückelten Riesenschlange (Kairo JE 68773) ${ }^{16}$. Die Schlange nimmt die gesamte Länge des Zauberstabs ein und könnte das früheste Bild des Götterfeindes Apophis als Riesenschlange darstellen.

\footnotetext{
${ }^{12}$ Newberry 1894: 36-38, Taf. 24 (Bd. I); Smith 1951: 321-332.

${ }^{13}$ Wreszinski 1927: Taf. 36; Wildung 1984: 95, Abb. 85; Davies, O’Connell 2009: 52, Abb. 1-2.

${ }^{14}$ Loprieno 2011: 55.

${ }^{15}$ Zur Bedeutung der Schlangen als feindliche Wesen vgl. Theis 2014: 794, s.v. hf $3 w$.

${ }^{16}$ Michałowski et al. 1938: 120, Nr. 600, Taf. 40.3.
} 
Zu den Feinden der Schlangen zählen alle auf den Zauberstäben abgebildeten numinosen Mächte, darunter nicht nur solche, die zu den natürlichen Feinden der Schlangen gehören (z.B. Ichneumon, Katze), sondern auch solche, die eigentlich Beutetiere der Schlangen sind, wie zum Beispiel der Frosch (Abb. 1b, 2a).

Die Tiermächte töten die Schlangen teilweise in aufgerichteter Haltung und auf den Hinterbeinen stehend. Große Schlangenfeinde sind der Löwe und das Nilpferd, die in aufgerichteter Haltung mit und ohne Messer Schlangen vernichten. Eine katzenartige Gottheit packt in aufgerichteter Haltung eine Schlange und enthauptet sie mit einem Messer (MMA 15.3.951.A, Jerusalem IAA 1948-1391). Fast alle auf den Zauberstäben abgebildeten Tiere sind als Schlangenfeinde einzustufen. Generell sind die Schlangenfeinde mit einem Messer ausgestattet. Auch die auf den Zauberstäben abgebildeten Gottheiten führen ein Messer, wie die auf einem Schrein liegende Kuh, die Doppelsphinx, der Doppelstier, der Pavian und das Seth-Tier. Selbst Fabelwesen zählen zu den Feinden der Schlangen, darunter der falkenköpfige Greif, der eine der am häufigsten abgebildeten Figuren der Zauberstäbe ist und mit seinem Falkenkopf Schlangen aufpickt (Abb. 1b) und der sogenannte „Schlangenhalspanther“, der mit den Zähnen seines Pantherkopfs die Schlangen zermalmt ${ }^{17}$.

Eine besonders aktive Schutzgottheit ist der besgestaltige Dämon mit dem Namen ‘h3 „Kämpfer“. Er packt mit jeder Hand meist eine einzelne Schlange (s.u. Abb. 1b, 2a-b, 3a-b), ausnahmsweise einmal auch zwei Schlangen (Abb. 1b). Auf dem Zauberstab Kairo CG 9438 scheint er ganz ungewöhnlich einmal eine Schlange und einen Skorpion in den Händen zu halten.

Beim Einfangen der Schlangen wird der besgestaltige Dämon häufig durch seine weibliche Partnerin unterstützt, die ebenfalls mit Schlangen in den Händen abgebildet ist (Manchester 1799), meist aber Eidechsen und Hasen hält (Abb. 2a, 3a) ${ }^{18}$. Der hundeköpfige Dämon des Zauberstabs Basel BSAe 991, der ebenfalls Eidechsen in der Hand hält, erinnert an den Eidechsenfänger der Vignette zu $T b 182^{19}$.

\section{DIE FEINDE IN MENSCHENGESTALT}

$\mathrm{Zu}$ den besonders gefährlichen Feinden, die vernichtet werden, zählen die Vertreter von Fremdvölkern, unter denen auf den gebogenen Zauberstäben des Mittleren Reiches und der Zweiten Zwischenzeit die Libyer und Asiaten aufgrund ihrer Tracht zweifelsfrei zu bestimmen sind. Wahrscheinlich gehören auch die Nubier zu den traditionellen Feinden der Götter der Zauberstäbe ${ }^{20}$.

\footnotetext{
${ }^{17}$ Eine ausführliche Aufstellung der auf den Zauberstäben abgebildeten Wesen kann aus Platzgründen nicht erfolgen. Hierzu ist vorläufig auf den Katalog Altenmüller 1965 (Bd. II) zu verweisen.

${ }^{18}$ Weingarten 2015: 181-189.

${ }^{19}$ Altenmüller 1986; Lucarelli 2012.

${ }^{20}$ Yamazaki 2003: 16; Borghouts 1970: 151; Koenig 1987: 105.
} 


\section{DER LIBYER}

Der Libyer wird auf dem Zauberstab Louvre E 3614 + MMA 26.7.1288 a-b (Abb. 2b) von einer hinter ihm sich aufrichtenden Löwengottheit gepackt ${ }^{21}$. Eine Fesselung ist nicht zu erkennen. Er nimmt eine ähnliche Haltung ein wie die Libyer der königlichen Denkmäler des Alten Reiches, die von Greifen und Sphinxen niedergetreten werden ${ }^{22}$. Seine stürzende Haltung gibt den hilflosen Zustand des Mannes an. Die Arme sind nach unten zum Boden geführt, das rechte Bein weit vom Körper weg gestreckt, das linke kniend angewinkelt.

Der zu Boden fallende Libyer ist in der libyschen Landestracht wiedergegeben, deren Details mit den Bildern der libyschen Gefangenen des Sahure aus dem Alten Reich weitgehend übereinstimmen ${ }^{23}$. Im Stürzen fallen die Haare in einer breiten Strähne nach hinten, der spitze Kinnbart weist leicht nach oben. Über der Brust sind zwei gekreuzte Schärpen $\mathrm{zu}$ erkennen. Ein hoher Perlenkragen und ein Halsband mit einer etwa bis zum Nabel herabhängenden Kordel schmücken Brust und Oberkörper. Die aus aufgezogenen runden und ovalen Perlen bestehende Kordel ist zwar nicht zu sehen, aber das untere konische Abschlussstück ist klar heraus gearbeitet. Um die Hüfte ist ein Gürtel gelegt, an dem seitlich eine halbrunde Schlaufe herabhängt und von dem vorne eine Phallustasche abgeht. Ein am Gürtel befestigter Tierschwanz, den man erwartet, ist nicht abgebildet.

Der Libyer des Zauberstabs Baltimore WAM 71510 (Abb. 1b) wird von hinten von einem Löwen angefallen. Der angreifende Löwe hockt auf den Hinterbeinen, um seinen Oberkörper sind Bänder gebunden, wie dies auch bei den Löwen der Thronpodeste des Alten Reiches (Sahure ${ }^{24}$, Neith ${ }^{25}$, Udjebten ${ }^{26}$ ) sowie des Mittleren und Neuen Reiches der Fall ist ${ }^{27}$. Er drückt mit der rechten Vordertatze den Feind nach unten, der durch die über der Brust sich kreuzenden Schärpen als Libyer identifiziert ist.

Ein weiteres Beispiel liefert der gebogene Zauberstab MMA 15.3.197 VS aus Lischt (Abb. 4a), auf dem ein Löwe zwei Feinde niedertritt. Der Löwe erweist sich durch die Schulterrosette als ein junger Löwe ${ }^{28}$. Einer der beiden Feinde ist an dem Kreuzband über der Brust und an der vollen Haarfülle als Libyer zu identifizieren. Die Ethnizität des in unnatürlichen Verrenkungen und mit gestrecktem Bein am Boden liegenden zweiten Feindes ist nicht klar. Der Löwe, der über den Feind hinweg schreitet, setzt die rechte Vordertatze auf den Oberschenkel des Libyers auf, dessen rechten Unterarm er mit seinem Maul packt und zum Teil bereits verschlungen hat.

Vermutlich ist auch der Feind des Zauberstabs London UC 15917, dessen Kopf auf der Vorder- und Rückseite des Zauberstabs von einem auf den Hinterbeinen aufgerichteten

${ }^{21}$ Quirke 2015: 199-200; zum Bildmotiv vgl. Schoske 1994: 391-401.

${ }^{22}$ Stockfisch 1996.

${ }^{23}$ Borchardt 1913: 12-13, Blatt 1 und 5; Wildung 2010: 185, Abb. 147.

${ }^{24}$ Awady 2009: Taf. 6.

${ }^{25}$ Jéquier 1933: Taf. 4-5.

${ }^{26}$ Jéquier 1928: 17, Abb. 13.

${ }^{27}$ Kuhlmann 1977: 77 mit Verweisen; Pongracz 1957: 213-220.

${ }^{28}$ Budde 2000. Die im Original vorhandene Schulterrosette ist in der Zeichnung leider nicht angegeben. 
Löwen abgerissen wird, ein Libyer ${ }^{29}$. Denn auf dem Körper des Gefangenen, der mit nach hinten gefesselten Armen abgebildet ist, sind die Umrisse der über der Brust gekreuzten Schärpe der Libyertracht in schwachen Linien zu erkennen. Allerdings stellt die Form des in Streifen nach unten fallenden Schurzes(?) den libyschen Identifikationsvorschlag in Frage.

Das Vorbild für das Motiv des Niedertretens der libyschen Feinde durch Tiermächte liefern ohne Zweifel die Szenen des Erschlagens der Feinde in den Pyramidenanlagen des Alten Reiches, wo der König als Sphinx oder Greif die libyschen und asiatischen Feinde niedertritt $^{30}$. Allerdings fällt in den monumentalen Szenen der königlichen Denkmäler nur dem Sphinx und dem Greifen die Rolle der Vernichtung der libyschen Feinde zu, während auf den Zauberstäben die Löwengottheit, dort mit einem nicht übersetzbaren Namen $h s m$ versehen (vgl. Abb. 4b) ${ }^{31}$, den Feind vernichtet. Möglicherweise ist die Löwengottheit mit „Mahes, dem Sohn der Bastet“" gleichzusetzen ${ }^{32}$.

\section{Der AsIATE}

Der asiatische Feind des Zauberstabs Louvre E 3614 + MMA 26.7.1288 a-b (Abb. 2b) ist wie der Libyer an seiner Landestracht zu identifizieren, für die die pilzförmige Frisur und der spitze Kinnbart charakteristisch sind ${ }^{33}$. Der in kniender Haltung und mit auf dem Rücken gefesselten Armen abgebildete Asiat wird frontal von der Nilpferdgottheit angegriffen, die sich vor ihm auf den Hinterbeinen hoch aufrichtet und im Begriff ist, ihn mit weit aufgerissenem Rachen zu zerfleischen. Die Nilpferdgottheit wird wie das Sternbild der astronomischen Deckenbilder des Neuen Reiches mit einem Krokodil auf dem Rücken abgebildet, vielleicht als Bild der Göttin Isis ${ }^{34}$.

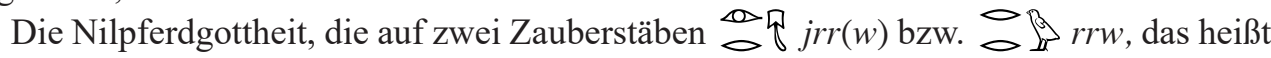
„Sau“, genannt ist ${ }^{35}$, tritt im königlichen Bereich als Kampfgottheit nicht hervor, sondern erfüllt soweit erkennbar hauptsächlich die Funktion einer Schutzgottheit. Ihre Schutzfunktion wird in einer ausführlichen Aretalogie auf dem Rückenpfeiler einer Nilpferdstatue des Louvre (Louvre E 25479) aus der 22. Dynastie hymnisch gepriesen, wo sie unter den Namen Reret, Thoeris und Ipet auftritt ${ }^{36}$.

\footnotetext{
${ }^{29}$ Altenmüller 1965: 66-67 (Bd. II); Legge 1905: 299-300, Abb. 47.

${ }^{30}$ Bates 1914: 122-127; Stockfisch 1996: 315-325; Schoske 1994: 365-401 (Bd. I) mit Parallelstellen. Im szenischen Umfeld der Pyramidenanlage und der Königsikonographie verkörpert der König in seiner Tiergestalt, wie die Beischrift zum Sphinx des Sahure (Borchardt 1913: Blatt 8 [Bd. II]) zeigt, ,Thot, den Herrn der Iuntiu“ und „Sopdu, den Herrn der Fremdländer“.

${ }^{31}$ Kopenhagen NM 7795; Kairo E.2007.04.58: Polz et al. 1999: 390.

${ }^{32}$ Habachi 1985: 93, Abb. 12 (Bd. I); Taf. 161c-163 (Bd. II); vgl. Žabkar 1975: 52-62; Wit 1978: $230-234$.

${ }^{33}$ Schiestl 2006: 173-185; s. auch Kanawati, Evans 2014: Taf. 129.

${ }^{34}$ Lieven 2000a: 24-26.

${ }^{35}$ Kopenhagen NM 7795 (jrrw); Kairo E.2007.04.58 (rrw): Polz et al. 1999: 390.

${ }^{36}$ Vandier 1962: 197-204; Jansen-Winkeln 2005: 140-146, Taf. 9-11; 2007: 92-93 (Nr. 14).
} 
Als Reret ist sie Schutzgottheit für den Neugeborenen.

(1) Ich bin die Sau (rrt), die angreift mit ihrem Gebrüll, die frisst, wenn sie sich nähert, laut an Stimme und brüllend an Geschrei, die den beschützt, der aus ihrem Leib kommt.

Als Thoeris ist sie Isis, die die Feinde des Horus vertreibt.

Ich bin Thoeris (t3-wrt) in ihrer Mächtigkeit,

die kämpft um ihren Besitz,

die die Feinde vertreibt von (2) ihrem Sohn Horus.

Als Ipet geht sie im Horizont gegen die Feinde des Allherrn vor.

Ich bin Ipet (jpt), die sich im Horizont befindet, und deren Messer den Allherrn beschützt, die Herrin des Schreckens, von vollkommener Gestalt, die den enthauptet, der gegen ihn (den Allherrn) rebelliert.

\section{DER NUBIER}

Ein nubischer Feind ist auf den Zauberstäben nicht mit Sicherheit zu identifizieren. Dennoch wird man annehmen dürfen, dass auch Nubier zu den Feinden zählen, die von den Schutzgottheiten der Zauberstäbe vernichtet werden ${ }^{37}$. Sollte der Vergleich des Zauberstabs München ÄS 2952 (Abb. 1a) mit dem Pektoral aus dem Grab der Prinzessin Merit (Kairo CG 52002, JE 30875) in Dahschur Bestand haben, was nicht sicher ist, müsste man zumindest auf dem Münchner Zauberstab mit einem nubischen Feind rechnen, weil auf dem Pektoral, das das gleiche Motiv wie der Zauberstab in München wiedergibt, die beiden Feinde jeweils durch das Inkarnat „rot" und „schwarz" als Asiat und als Nubier unterschieden $\operatorname{sind}^{38}$. Die beiden Feinde des Zauberstabs werden von Löwen niedergetreten und nicht von Greifen, wie die Königsikonographie erfordert hätte.

\section{NiCHT IDENTIFIZIERTE MENSCHLICHE FEINDE}

Menschliche Feinde, die auf den Zauberstäben ohne ein charakteristisches ethnisches Merkmal wiedergegeben sind, lassen sich nur schwer einordnen. Dazu gehören die menschlichen Feinde, deren abgetrennter Kopf vor einem Krokodil liegt (Berlin ÄM 14207, Kairo CG 9435, London UC 16384, MMA 32.8.4 Rs). Möglicherweise handelt es sich bei ihnen um Rebellen, die aufgrund ihrer Haartracht auch Ägypter sein könnten.

a) Zauberstab MMA 15.3.197 VS (Abb. 4a). Der von hinten von einem Nilpferd angegriffene Feind kann nicht mit Sicherheit einer bestimmten Ethnie zugeordnet werden. Die mit einem Messer bewehrte Nilpferdgottheit hat sich auf den Hinterbeinen aufgerichtet

\footnotetext{
${ }^{37}$ Zur Rolle von Nubien in der altägyptischen Magie vgl. Koenig 1987.

${ }^{38}$ Vernier 1927: Taf. 1 (Kairo CG 52002); PM III.2, 884.
} 
und packt den rechten Arm des vor ihr zusammenbrechenden menschlichen Feindes, der sich im Sturz mit dem linken Arm am Boden abstützt. Möglicherweise steht sein Tod kurz bevor, da ein Blutstrahl aus seiner Stirn austritt.

b) Zauberstab Berlin ÄM 14207 RS (Abb. 2a). Ein auf den Hinterbeinen aufgerichtetes katzenartiges Tier enthauptet mit einem Messer, das es mit der Pfote festhält, einen menschlichen Feind. Aus der Stirn des vom Körper abgetrennten Kopfes fließt Blut.

c) Zauberstab MMA 15.3.197 RS (Abb. 3a). Ein mit nach hinten gefesselten Armen am Boden kniender Feind wird von einem katzenartigen Tier angefallen. Das Tier steht aufrecht auf den Hinterbeinen und tritt auf den Oberschenkel des Feindes. Es legt die Vordertatzen auf die Schulter des Gefesselten und zerfleischt den Kopf.

d) Zauberstab Louvre E 20611 (Abb. 3b). Ein am Boden hockendes katzenartiges Tier, das eine Schlange im Maul und ein Messer zwischen den Vorderbeinen hat, fixiert einen am Boden knienden gefesselten Feind. Es scheint sich um einen Rebellen zu handeln, dessen Hinrichtung unmittelbar bevorsteht.

Das auf den Zauberstäben beim Zerfleischen von menschlichen Feinden und von Schlangenfeinden gezeigte Bild der Katzengottheit könnte ein Bild des „großen Katers“ von Heliopolis sein, der in $T b 17^{39}$ als strafende Gottheit den Götterfeind Apophis zerstükkelt und in dieser Funktion mit Re, dem Herrn von Heliopolis, gleichgesetzt wird ${ }^{40}$. Die Identifikation der Katzengottheit mit Re, möglicherweise in Abhängigkeit von $T b$ 17, ist im Neuen Reich durch mehrere Denksteine aus Deir el-Medineh bezeugt ${ }^{41}$. Auch bei den Zauberstäben des Mittleren Reiches ist eine solche Identifikation denkbar, da durch das Vernichten des Feindes die Schutzfunktion der abgebildeten Gottheit herausgestellt wird. Letzte Sicherheit lässt sich aber wegen fehlender Beischriften nicht gewinnen ${ }^{42}$. Da die Katze auf einem Zauberstab wie die Göttin Bastet mit einem Halsschmuck versehen ist ${ }^{43}$, könnte es sich auch um ein Bild der Göttin Bastet handeln oder um eine andere Gottheit, die in ähnlicher Funktion wie der ,große Kater“ von Heliopolis auftritt.

\section{AUSWERTUNG DER FEINDBILDER}

\section{DiE KÖNIGSIKONOGRAPHIE ALS VORBILD}

Die Untersuchung hat ergeben, dass bei den auf den Zauberstäben des Mittleren Reiches und der Zweiten Zwischenzeit abgebildeten Feinden im Wesentlichen zwischen Schlangenfeinden und menschlichen Feinde zu trennen ist. Die Schlangenfeinde werden von den

\footnotetext{
${ }^{39}$ Hornung 1979: Kap 17, Vers 192-195.

${ }^{40}$ Corteggiani 1995: 144-151; Karlshausen 1991: 104-105.

${ }^{41}$ Servajean 2002: 355-370.

${ }^{42}$ Vgl. auch Hornung 1963: 120, Nr. 495 (Bd. I); 128 (Bd. II).

${ }^{43}$ Kofler A 101.
} 
Mächten der Zauberstäbe vertrieben, gepackt, zerbissen oder mit dem Messer zerfleischt. Ihre Vernichtung symbolisiert den Sieg der Gottheiten über feindliche Mächte. Das Ereignis liefert den Präzedenzfall für die reale Abwehr von Schlangen, die in der Nacht oder bei Tag Mutter und Kind in Gefahr bringen.

Im Unterschied zu den Schlangen, die fast ausnahmslos von allen Gottheiten der Zauberstäbe überwunden werden können, werden die Feinde in Menschengestalt nur von einigen wenigen ausgewählten Gottheiten bekämpft, und zwar von der Löwengottheit, der Nilpferdgottheit und der Katzengottheit. Die von den Tiermächten bekämpften Feinde haben die Gestalt von Libyern, Asiaten, vielleicht auch von Nubiern. Zu den gefährlichen Feinden zählen möglicherweise auch Ägypter, die als Rebellen abgestempelt sind.

Die auf den Zauberstäben verwendete Ikonographie des Niederschlagens der menschlichen Feinde orientiert sich formal an der politischen Ikonographie des Königtums, in der sich der König beim Niederschlagen der Feinde in der göttlichen Gestalt von Stier, Löwe, Greif oder Sphinx präsentieren kann. Der König, der in Gestalt des Greifs die Feinde niedertritt ${ }^{44}$, wird in den königlichen Monumenten mit Thot oder Sopdu gleichgesetzt. Dergleichen Identifikationen lassen sich bei den Schutzgottheiten der Zauberstäbe aber nicht feststellen. Die Tiermächte der Zauberstäbe, die beim Niederschlagen der menschlichen Feinde abgebildet sind, übernehmen zwar formal die Haltungen der politischen Ikonographie des Königtums, sind aber inhaltlich auf ihr Wesen als Tiermacht beschränkt.

\section{Der Kampf des Sonnengottes Gegen die Mächte des Chaos}

In den Sonnenhymnen des Neuen Reiches wird der Sonnenaufgang als Beginn der Herrschaft des Sonnengottes gepriesen und der tägliche Sonnenlauf mit dem Königtum des Sonnengottes verglichen. Doch die Freude über den Herrschaftsantritt des Sonnengottes provoziert ständig auch Widerstand und Rebellion, mit dem Ziel, den Sonnenlauf anzuhalten. Der täglich neu entfachte Aufstand gegen den Sonnengott muss daher immer wieder neu niedergeschlagen werden, wird aber auch niemals vollständig beendet. Der Kampf, der bei Sonnenaufgang gegen den Erzfeind der Sonne gerichtet ist, wird in den Sonnenhymnen des Neuen Reiches beschrieben ${ }^{45}$, über den nächtlichen Kampf des Sonnengottes berichten ausführlich die etwa gleichzeitigen Texte des Amduat (7. Stunde) ${ }^{46}$.

Dieser ständige Kampf der Gefolgsleute des Sonnengottes gegen die Rebellen ist Vorbild und gleichzeitig auch Gegenstand des Bildprogramms der Zauberstäbe. Die Nilpferdgottheit, die Löwengottheit und die katzenartige Gottheit sind Vorkämpfer in diesem Kampf, den sie sowohl in der Tagesphase als auch in der Nachtphase des Sonnenlaufs führen. Der Kampf ist repetitiv und wiederholt sich an jedem Tag aufs Neue.

In Erkenntnis dieses alltäglichen Kampfgeschehens am Himmel darf man dann auch annehmen, dass es sich bei den mächtigen Tieren der Zauberstäbe um „himmlische“

\footnotetext{
${ }^{44}$ Borchardt 1913: Blatt 8.

${ }^{45}$ Assmann 1999: 34-35.

${ }^{46}$ Hornung 1963: 139-140 (Bd. II).
} 
Wesen handelt, die sich entlang der Sonnenbahn bewegen und ihren jeweils spezifischen Beitrag zur Vertreibung oder Vernichtung der auf den Zauberstäben abgebildeten Feinde im magischen Diskurs leisten.

\section{Sterne und Dekane im Gefolge des Sonnengottes}

Die auffallende Gestalt der Nilpferdgottheit mit einem Krokodil auf dem Rücken, die auf dem Zauberstab Louvre E 3614 + MMA 26.7.1288 a-b (Abb. 2b; vgl. Basel BSAa 991, MMA 30.8.218) zu sehen ist, legt die Annahme nahe, dass es sich bei der Nilpferdgottheit um ein Sternbild handelt. Ähnliche Bilder der Nilpferdgottheit sind von den astronomischen Deckenbildern des Neuen Reiches und der Spätzeit gut bekannt ${ }^{47}$. Man ist daher berechtigt zu vermuten, dass die anderen Gottheiten der Zauberstäbe ebenfalls Sternbilder oder Dekane sein könnten. Ihr Handeln wird in einer Architravinschrift des griechisch-römischen Tempels von Esna (Esna 400) treffend beschrieben ${ }^{48}$. Der späte Text, der in Teilen Vorgänger im Pap. Leiden I 346 I, 4-6 aus der 18. Dynastie besitzt, lautet in der Übersetzung von J.F. Quack ${ }^{49}$ :

Heil euch, lebende Götter,

Dekane in der Nekropole,

die in der Dunkelheit leuchten,

die die Stunden terminieren bei ihrem Kommen im Osten,

die ankündigen, was geschieht,

die nach ihrem Wunsch am Leben erhalten und Frevler töten,

die im Himmel erscheinen, die die Länder mit Feuer beschießen,

bei deren Hervorkommen jedermann zittert,

deren Zeiten die Astronomen beobachten.

Seid gegrüßt, Kinder des Re, die bei seinem Untergang erscheinen,

Neunheit der ersten Urzeitlichen.

Sie folgen ihm (Re) Tag für Tag,

die lebenden Seelen der Götter, Tag für Tag.

Sie ziehen umher als Auge des Re,

Boten in den Städten und Gauen,

die Pfeile schießen mit ihren Mündern gegen den, den sie von fern sehen.

Die täglich funkelnden Sterne, die ihren Herrn retten

vor allen üblen Dingen, vor dem Schießen der Sterngötter,

an diesem Tag, in diesem (Halb-)Monat, in diesem Monat, in diesem Jahr,

in all ihren Stunden bis heute.

\footnotetext{
${ }^{47}$ Neugebauer, Parker 1969: 189-190.

${ }^{48}$ Sauneron 1969: 9-10; Schott 1936: 16; Quack 1995: 100; Lieven 2000a: 42-55.

${ }^{49}$ Quack 1995: 100-101.
} 
Chnumis ist ausgerüstet an der Spitze der „, Göttlichen “ bei ihrem Durchzug.

Die Dekane, die lebenden, verrichten ihre Tätigkeit in der Nacht,

um hinter Orion und Sirius zu kulminieren,

wenn er in die Unterwelt des großen Gottes im Westen hinabtaucht, um ihm zu folgen, wenn er ermattet ist, bis in Ewigkeit, Tag für Tag.

Der Text beschreibt in seinem Anfangsteil ausführlich das Wirken der Dekane, als Schicksalsmächte und als Zeitdeterminanten. Sie sind Wesen, die in der Dunkelheit leuchten, die die Stunden terminieren bei ihrem Kommen im Osten, die ankündigen, was geschieht.

Der zweite Abschnitt des Esna-Textes, in dem auf die Rolle der Dekane im Gefolge des Sonnengottes näher eingegangen wird, hat eine partielle Parallele in dem aus der 18 . Dynastie stammenden Pap. Leiden I 346 I, 3-6 $6^{50}$. Im Text des Neuen Reiches treten die h3jtjw-Dämonen an die Stelle der Dekane des Esna-Textes: Seid gegrüßt, o ihr Götter, Messerdämonen (h3ijw), Vorhut der Sachmet, die aus dem Auge des Re hervorkommen, Boten, die die Gaue durchziehen, die Gemetzel anrichten, die Unruhe stiften, die das Land durcheilen und ihre Pfeile mit ihrem Maul verschießen gegen den, den sie von Ferne sehen.

Die Möglichkeit, die ḩ3tjw-Dämonen des Leidener Papyrus mit den Dekanen des EsnaTextes gleichzusetzen, ist wegen der großen Zeitdifferenz stark eingeschränkt. Doch weist J.F. Quack zu Recht darauf hin, dass die Rolle der ḩ3tjw als Dekangottheiten und Sternengötter sich auch aus anderen Texten erschließen lässt, wo sie - wie in den Pyramidentexten und Sargtexten - komplementär zu den „Sternen die nicht untergehen“ (jhmw sk) in einer Position auftreten, die in anderen religiösen Texten die „Sterne, die nicht ermatten“ (jhmw $w r \underline{d}$ ) einnehmen ${ }^{51}$. Man darf daher vermuten, dass auch auf den Zauberstäben die Götter der Götterprozessionen, die von ihrem Wesen her den $h 3$ tjw-Dämonen gleichen ${ }^{52}$, die Rolle von Dekangottheiten übernehmen ${ }^{53}$.

\section{Der GReIF Der ZauberstäBe IN DER Rolle eines DeKans?}

Ein Zauberstab aus Theben (Kairo E. 2007.04.58) scheint die These zu bestätigen, dass sich unter den Mächten der Götterprozessionen der Zauberstäbe des Mittleren Reiches und der Zweiten Zwischenzeit auch Dekane befinden (Abb. 4b). Die Götterprozession dieses thebanischen Zauberstabs besteht aus Gottheiten, von denen viele mit einer Beischrift versehen sind und Eigennamen tragen ${ }^{54}$. Dazu gehören von rechts nach links die Nilpferdgottheit $r r w$, der besgestaltige Dämon $` h 3$, der Greif mit einem schwer zu entziffernden Namen und die Löwengottheit ḥsm. Leider ist der Name des Greifs nur unvollständig erhalten, so dass sich seit der Entdeckung des Zauberstabs im Jahre 1999 zahlreichen Spekulationen um

\footnotetext{
${ }^{50}$ Bommas 1999: 34-41.

${ }^{51}$ Quack 1997: 283-284.

${ }^{52}$ Leitz 1994: 256-257.

${ }^{53} \mathrm{Zu}$ den h3tjw-Dämonen existiert eine umfangreiche Literatur, vgl. Leitz 1994: 244-255; Bommas 1999: 35-37; Lieven 2000a: 50-55; LGG V, 635-637.

${ }^{54}$ Polz et al. 1999: 395-398.
} 
den Namen gebildet haben, die alle nicht voll befriedigen und daher zu Recht angezweifelt werden $^{55}$. Die bisherigen Lesungsvorschläge sind:

(1) $\bigcirc \circ \backslash$ 핖 tptj jdbwj: ,Erster der beiden Ufer“" oder tptj $t 3 w j$,EErster der Beiden Länder“56.

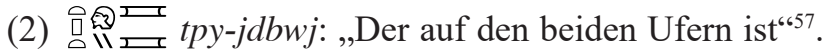

(3) 1 jn-hrt: „Onuris ${ }^{658}$.

Ein neuer Lesungsversuch, der, weil vom Foto ausgehend ${ }^{59}$, zugegebenermaßen ebenfalls

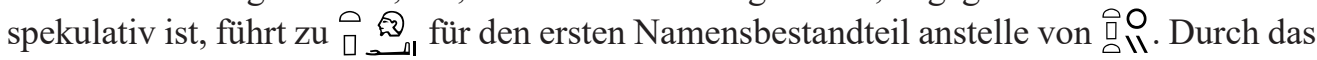
neu gewonnene nomen regens tpj- ${ }^{\complement}[\ldots]$ scheint eine Deutung des Greifs als Dekangottheit möglich zu sein, denn eine Vielzahl von Dekannamen beginnt mit tpj- ${ }^{-}$, womit üblicherweise der ,Vorläufer (eines Sterns)“ bezeichnet ist ${ }^{60}$. Die auf $t p j$-` folgenden Zeichenspuren sind auf dem Foto nicht mehr zu identifizieren, die vorgeschlagene Lesung wenig überzeugend $^{61}$. Für eine Ergänzung steht möglicherweise nur ein einziges Schriftquadrat zur Verfügung, so dass als eine wahrscheinliche Ergänzung von tpj-` [spdt] ,Vorläufer [der Sothis“] oder eine ähnlich Ergänzung in Betracht kommt, was aber am Foto der Grabungsberichts nicht entschieden werden kann und noch am Original zu überprüfen wäre.

In diesen astralen Zusammenhang des Greifbilds passt eine Beischrift zum Bild des Sphinx-Gottes Tutu auf einem zweiteiligen astrologischen Tablett, das in Grand (Vogesen) gefunden wurde und aus dem 2. Jahrhundert AD stammt ${ }^{62}$. Die Sphinxfigur, deren Ikonographie eine Gleichsetzung des Sphinx mit dem Gott Tutu erlaubt ${ }^{63}$, ist als dritter Dekan des Tierkreiszeichens Gemini auf den Seiten A und B des Tabletts abgebildet und steht unmittelbar vor Sothis im ersten Feld des Tierkreiszeichens Krebs. Auf dem Diptychon A des Tabletts steht beim Sphinx die Beischrift $\tau \varepsilon \pi \imath \sigma \mu v o$ und auf dem Diptychon B die Beischrift $\tau \varepsilon \pi \imath \sigma \mu о \theta$. Die Namen sind, wie O.E. Kaper zeigt, zwar verderbt, aber zweifelsfrei mit den aus anderen Quellen bekannten Dekannamen $\tau \varepsilon \pi \imath \sigma \omega \theta$ (Hermes Trismegistos) und $\tau \varepsilon \pi ı \varsigma$ (Firmicus Maternus) zu verbinden ${ }^{64}$, der griechischen Entsprechung des alten ägyptischen Dekannamens tpj-` spdt „Vorläufer der Sothis“.

Durch den Umstand, dass der Dekan Gem-3 auf dem Tablett aus Grand die Gestalt eines Sphinx als Verkörperung des Gottes Tutu aufweist und dieser durch seinen Namen $\tau \varepsilon \pi 1 \sigma \omega \theta$

${ }^{55}$ Gerke 2014: 109-110.

${ }^{56}$ Polz et al. 1999: 392, 396.

${ }^{57}$ LGG VII, 388.

${ }^{58}$ Altenmüller 2013: 11-28.

${ }^{59}$ Leider konnte das Original im Museum von Kairo nicht eingesehen werden.

${ }^{60}$ Neugebauer, Parker 1960: 2-3; 1969: 157-166 (Bd. I).

${ }^{61}$ Polz et al. 1999: 392.

${ }^{62}$ Willems, Clarysse (Hrsg.) 2000: 135-136, Abb. 62.

${ }^{63}$ Kaper 2003: 225-226 (M-19/20) mit Abbildung.

${ }^{64}$ Kaper 2003: 70-71, 225-226. Die Sphinxfigur wird einer sog. „Dritten Reihe“ von Dekanen zugeordnet, die Lieven 2000b: 21-36 gegen die Dekanen der Familien „Sethos I B“ und „Tanis“ abgrenzt. In der Sethos I B-Familie hat der Dekan die Gestalt einer aufrecht stehenden Schlange mit Armen, deren Hände $n w$-Töpfe halten: Neugebauer, Parker 1969: 139 (Bd. I), in einer „Gemischten Gruppe“ ist der Dekan mit Menschenkopf und Stern abgebildet: Neugebauer, Parker 1969: 152 (Bd. I). 
(Hermes Trismegistos) als der Dekan tpj-` spdt bestimmt werden kann, ist die Ergänzung des Namens des Greifs des Zauberstabs aus Theben als tpj- ${ }^{-}[s p d t]$ nicht unwahrscheinlich. Denn die Sphinxgestalt des spätzeitlichen Gottes Tutu hat vermutlich ihren Ursprung im Greif des Mittleren Reiches, wie sich aus einigen Merkmalen der Ikonographie des Gottes ergibt, zu denen die Flügel am Tierkörper und der Schwanz in Gestalt einer Kobra gehören; beide derartig ausgezeichnete Greifgestalten sind auf dem Zauberstab Baltimore WAM 71510 (Abb. 1b) und dem „magic rod“ Hannover, Kestner Museum 1949.350 zu beobachten ${ }^{65}$.

Mit der möglichen Identifizierung des Greifs als Dekan tpj-` [spdt] gewinnt die Vermutung Substanz, dass die Gottheiten der Zauberstäbe zu den Dekanen gehören und als Dekane den Sonnengott gegen seine Feinde verteidigen und schützen.

\section{ZUSAMMENFASSUNG}

Die auf den Zauberstäben des Mittleren Reiches und der Zweiten Zwischenzeit abgebildeten Feinde besitzen die Gestalt von Schlangen, von Asiaten, Libyern und wahrscheinlich auch von Nubiern sowie von innerägyptischen Rebellen, die gegen den Sonnengott und gegen die Weltordnung rebellieren. Die Analyse der Feindbilder und der die Feinde bekämpfenden Gottheiten ergibt, dass die überragenden Schutzgottheiten der Zauberstäbe die Nilpferdgottheit ( $r r w)$, die Löwengottheit ( $h \mathrm{sm}$ ) und die Katzengottheit (ohne Namen) sind, die als einzige die menschlichen Feinde attackieren und vernichten. Ihr jeweiliges theologisches Äquivalent ist schwer zu bestimmen, da Aussagen dazu fehlen. Im Ergebnis lässt sich nur vermuten, dass die Nilpferdgottheit in der Rolle der Göttin Ipet auftritt, die (männliche) Löwengottheit die Rolle des Mahes, des Sohnes der Sachmet respektive der Bastet übernimmt, und dass die Katzengottheit wohl nicht den Sonnengott Re darstellt, sondern eine andere Gottheit, vielleicht Bastet, die die $h 3$ tjww-Dämonen befehligt oder einen ihrer Verwandten.

$\mathrm{Zu}$ den Schutzgottheiten der Zauberstäbe zählen ohne Zweifel die Gottheiten des Himmels, zu denen die Sternbilder gehören (z.B. die Nilpferdgottheit), sowie die Dekangottheiten, die unter dem Oberbegriff der ḩ3tjw „Messerdämonen“ erfasst sind, ohne dass allerdings möglich wäre, in jedem Fall einen einzelnen Dekan namentlich zu benennen. Eine große Ausnahme bildet der Greif, der auf einem Zauberstab aus Theben, sofern die Ergänzung richtig ist, den Dekannamen ,Vorläufer [der Sothis]“ (tpj-` [spdt]) trägt. Dieser durch das Bild des Greifs abgebildete Dekan spielt im Mittleren Reich eine bedeutende Rolle an den Epagomenen und an Neujahr ${ }^{66}$, an dem im idealen Kalender die Göttin Sothis nach einer Periode der Unsichtbarkeit wieder erscheint und an dem der Geburtstag des Sonnengottes liegt, zu dessen Schutz die Götter der Zauberstäbe in einem großen Kampfgeschehen antreten. Das Bekämpfen der Feinde dürfte allerdings zeitlich nicht auf die Tage vom Jahresende beschränkt sein, da die Götter der Zauberstäbe „,bei Nacht und bei Tag“ (vgl. Abb. 4a) ihren Schutz ausbreiten.

\footnotetext{
${ }^{65}$ Gerke 2014: 111.

${ }^{66}$ Leitz 1995: 101-102.
} 


\section{Danksagungen}

Für wertvolles Bildmaterial danke ich Fred Vink, für entscheidende Hinweise einem ungenannten Referenten.

\section{Literaturverzeichnis}

Altenmüller, H. 1965: Die Apotropaia und die Götter Mittelägyptens. Eine typologische und religionsgeschichtliche Untersuchung der sog. „Zaubermesser“ des Mittleren Reichs, München

Altenmüller, H. 1983: Ein Zaubermesser aus Tübingen, $W d O$ 14, 30-45

Altenmüller, H. 1986: Ein Zaubermesser des Mittleren Reiches, SAK 13, 1-27

Altenmüller, H. 2013: Der rettende Greif: Zu den Bildern des Greifs auf den sog. Zaubermessern des Mittleren Reiches, [in:] Floßmann-Schütze, M.C., Goecke-Bauer, M., Hoffmann, F., Hutterer, A., Schlüter, K., Schütze, A., Ullmann, M. (Hrsg.), Kleine Götter - große Götter: Festschrift für Dieter Kessler zum 65. Geburtstag, Vaterstetten, 11-27

Altenmüller, H. 2015: Zur Bedeutung der Fabeltiere von Beni Hassan, [in:] Massiera, M., Mathieu, B., Rouffet, F. (Hrsg.), Apprivoiser le sauvage / Taming the Wild, Cahiers "Égypte Nilotique et Méditerranéenne » 11, Montpellier, 19-30

Arnold, D., Settgast, J. 1966: Zweiter Vorbericht über die vom Deutschen Archäologischen Institut Kairo im Asasif unternommenen Arbeiten (3. Kampagne), MDAIK 21, 72-94 Assmann, J. 1999: Ägyptische Hymnen und Gebete: übersetzt, kommentiert und eingeleitet, 2., verbesserte und erweiterte Auflage, $O B O$ (Sonderband), Freiburg, Schweiz-Göttingen

Awady, T. El- 2009: Sahure - The Pyramid Causeway: History and Decoration Program in the Old Kingdom, Excavations of the Czech Institute of Egyptology, Abusir XVI, Prague

Bates, O. 1914: The Eastern Libyans: An Essay, London

Bissing, F.W. von 1934: Ägyptische Kunstgeschichte von den ältesten Zeiten bis auf die Eroberung durch die Araber: Systematisches Handbuch, Berlin

Bommas, M. 1999: Die Mythisierung der Zeit: Die beiden Bücher über die altägyptischen Schalttage des magischen pLeiden I 346, GOF IV, Reihe Ägypten 37, Wiesbaden

Borchardt, L. 1913: Das Grabdenkmal des Königs Scajḥu-Rec ${ }^{c}$, Band II: Die Wandbilder, WVDOG 26, Leipzig

Borghouts, J.F. 1970: The magical texts of papyrus Leiden I 348, OMRO 51, Leiden

Budde, D. 2000: Zur Symbolik der sogenannten Schulterrosette bei Löwendarstellungen, ZÄS 127/2, 116-135

Corteggiani, J.-P. 1995: La « butte de la Décollation », à Héliopolis, BIFAO 95, 141-151

Davies, N. de G. 1943: The Tomb of Rekh-mi-rē‘ at Thebes, PMMA Egyptian Expedition XI, New York

Davies, W.V., O'Connell, E.R. 2009: The British Museum Expedition to Elkab and Hagr Edfu, 2009, BMSAES 14, 51-72 
Gerke, S. 2014: Der altägyptische Greif: von der Vielfalt eines „Fabeltiers“, SAK Beihefte 15, Hamburg

Gnirs, A.M. 2009: Nilpferdstosszähne und Schlangenstäbe: Zu den magischen Geräten des so genannten Ramesseumsfundes, [in:] Kessler, D., Schulz, R., Ullmann, M., Verbovsek, A., Wimmer, S.J. (Hrsg.), Texte - Theben - Tonfragmente: Festschrift für Günter Burkard, $\ddot{A} A T$ 76, Wiesbaden, 128-156

Habachi, L. 1985: The Sanctuary of Heqaib, Elephantine IV, ArchVer 33, Mainz a/Rhein Hornung, E. 1963: Das Amduat: Die Schrift des verborgenen Raumes, $\ddot{A g A b h} 7$, Wiesbaden Hornung, E. 1979: Das Totenbuch der Ägypter, Zürich-München

Jansen-Winkeln, K. 2005: Vier Denkmäler einer thebanischen Offiziersfamilie der 22. Dynastie, $S A K 33,125-146$

Jansen-Winkeln, K. 2007: Inschriften der Spätzeit II: Die 22.-24. Dynastie, Wiesbaden Jéquier, G. 1928: La pyramide d'Oudjebten. Fouilles à Saqqarah, Le Caire

Jéquier, G. 1933: Les pyramides des reines Neit et Apouit. Fouilles à Saqqarah, Le Caire Kanawati, N., Evans, L. 2014: Beni Hassan I: The Tomb of Khnumhotep II, The Australian Centre for Egyptology: Reports 36, Oxford

Kaper, O.E. 2003: The Egyptian God Tutu: a study of the sphinx-god and master of demons with a corpus of monuments, OLA 119, Leuven

Karlshausen, C. 1991: Le chat dans la mythologie: les démons-chats, [in:] Delvaux, L., Warmenbol, E. (Hrsg.), Les divins chats d'Égypte: un air subtil, un dangereux parfum, Leuven, 101-107

Koenig, Y. 1987: La Nubie dans les textes magiques «L'inquiétante étrangeté », $R d E 38,105-110$ Kuhlmann, K.P. 1977: Der Thron im alten Ägypten: Untersuchungen zu Semantik, Ikonographie und Symbolik eines Herrschaftszeichens, $A D A I K$ 10, Glückstadt

Legge, F. 1905: The magic ivories of the Middle Empire, PSBA 27, 130-152, 297-303

Legge, F. 1906: The magic ivories of the Middle Empire, PSBA 28, 159-170

Leitz, C. 1994: Tagewählerei: das Buch ḩ3t nḥh ph.wy $\underline{d} t$ und verwandte Texte, $\ddot{A} g A b h$ 55, Wiesbaden

Leitz, C. 1995: Altägyptische Sternuhren, OLA 62, Leuven

Lieven, A. von 2000a: Der Himmel über Esna: eine Fallstudie zur religiösen Astronomie in Ägypten am Beispiel der kosmologischen Decken- und Architravinschriften im Tempel von Esna, $\ddot{A g} A b h$ 64, Wiesbaden

Lieven, A. von 2000b: Die dritte Reihe der Dekane oder Tradition und Innovation in der spätägyptischen Religion, Archiv für Religionsgeschichte 2, 21-36

Loprieno, A. 2011: Literatur und Religion in Ägypten: ein Antagonismus?, [in:] Fischer-Elfert, H.-W., Richter, T.S. (Hrsg.), Religion und Literatur im Alten Ägypten: Ein Symposium zu Ehren von Elke Blumenthal, AAWL 81/5, Leipzig, 47-60

Lucarelli, R. 2012: The so-called vignette of Spell 182 of the Book of the Dead, [in:] Lucarelli, R., Müller-Roth, M., Wüthrich, A. (Hrsg.), Herausgehen am Tage: Gesammelte Schriften zum altägyptischen Totenbuch, SAT 17, Wiesbaden, 79-91

Michałowski, K., Linage, J. de, Manteuffel, J., Sainte Fare Garnot, J. 1938: Tell Edfou 1938. Fouilles franco-polonaises: Rapports II, Le Caire 
Neugebauer, O., Parker, R.A. 1960: Egyptian astronomical texts I: The early decans, Brown Egyptological Studies 3, London

Neugebauer, O., Parker, R.A. 1969: Egyptian astronomical texts III: Decans, planets, constellations and zodiacs, Brown Egyptological Studies 6, London

Newberry, P.E. 1894: El Bersheh, ASEg 3, London

Pinch, G. 1994: Magic in ancient Egypt, London

Polz, D., Gordon, W.E., Nerlich, A., Piccato, A., Rummel, U., Seiler, A., Voss, S. 1999: Bericht über die 6., 7. und 8. Grabungskampagne in der Nekropole von Dra' Abu el-Naga/Theben-West, MDAIK 55, 343-410

Pongracz, M. 1957: Löwendarstellungen an Podesten der Königsthrone, MDAIK 15, 213-220

Quack, J.F. 1995: Dekane und Gliedervergottung: altägyptische Traditionen im Apokryphon Johannis, JAC 38, 97-122

Quack, J.F. 1997: Rezension Leitz, C., Tagewählerei: das Buch ḩ3t nḥh ph.wy $\underline{d} t$ und verwandte Texte, LingAeg 5, 277-287

Quirke, S. 2005: Lahun: a town in Egypt 1800 BC, and the history of its landscape, London

Quirke, S. 2015: Apotropaic Wand, [in:] Oppenheim, A., Arnold, Do., Arnold, Di., Yamamoto, K. (Hrsg.), Ancient Egypt transformed: the Middle Kingdom, New Haven-London, 199-200

Sauneron, S. 1969: Le temple d'Esna (Nos. 399-472). Texte, PIFAO, Esna IV ${ }^{1}$, Le Caire

Schiestl, R. 2006: The statue of an Asiatic man from Tell el-Dab`a, Egypt, ÄgLev 16, 173-185

Schoske, S. 1994: Das Erschlagen der Feinde: Ikonographie und Stilistik der Feindvernichtung im alten Ägypten, Ann Arbor

Schott, S. 1936: Die altägyptischen Dekane, [in:] Gundel, W. (Hrsg.), Dekane und Dekansternbilder: ein Beitrag zur Geschichte der Sternbilder der Kulturvölker, Studien der Bibliothek Warburg 19, Hamburg

Servajean, F. 2002: À propos d'une hirondelle et de quelques chats à Deir al-Médîna, BIFAO 102, 353-370

Smith, W.S. 1951: Paintings of the Egyptian Middle Kingdom at Bersheh, AJA 55/4, 321-332

Stockfisch, D. 1996: Bemerkungen zur sog. ,libyschen Familie', [in:] Schade-Busch, M. (Hrsg.), Wege öffnen: Festschrift für Rolf Gundlach zum 65. Geburtstag, $\ddot{A} A T$ 35, Wiesbaden, 315-325

Terrace, E.L.B. 1967: Egyptian Paintings of the Middle Kingdom. The Tomb of Djehutynekht, New York

Theis, C. 2014: Magie und Raum: Der magische Schutz ausgewählter Räume im alten Ägypten nebst einem Vergleich zu angrenzenden Kulturbereichen, Orientalische Religionen in der Antike. Ägypten, Israel, Alter Orient 13, Tübingen

Vandier, J. 1962: Une statuette de Touéris, RevLouvre 12, 197-204

Vandier, J. 1969: Nouvelles acquisitions: Musée du Louvre, Département des antiquités égyptiennes, RevLouvre 19, 43-54 
Vernier, É. 1927: Bijoux et orfèvreries. Catalogue Général des Antiquités Égyptiennes du Musée du Caire N ${ }^{\text {os }}$ CG 52001-53855, Le Caire

Weingarten, J. 2015: The Arrival of Bes[et] on Middle-Minoan Crete, [in:] Mynářová, J., Onderka, P., Pavúk, P. (Hrsg.), There and Back Again - the Crossroads II. Proceedings of an International Conference Held in Prague, September 15-18, 2014, Prague, 181-198

Wildung, D. 1984: Sesostris und Amenemhet: Ägypten im Mittleren Reich, München

Wildung, D. 2010: Die Berliner Reliefs aus der Pyramidenanlage des Sahure, [in:] Brinkmann, V. (Hrsg.), Sahure: Tod und Leben eines grossen Pharao. Eine Ausstellung der Liebieghaus Skulpturensammlung, Frankfurt am Main, 24. Juni bis 28. November 2010, Frankfurt-München, 183-194

Willems, H., Clarysse, W. (Hrsg.) 2000: Les empereurs du Nil, Leuven-Paris

Wit, C. de 1978: Le rôle et le sens du lion dans l'Égypte ancienne, Luxor

Wreszinski, W. 1927: Bericht über die photographische Expedition von Kairo bis Wadi Halfa zwecks Abschluß der Materialsammlung für meinen Atlas zur altägyptischen Kulturgeschichte, SKGG, Geisteswissenschaftliche Klasse 4/2, Halle

Yamazaki, N. 2003: Zaubersprüche für Mutter und Kind: Papyrus Berlin 3027, Achet Schriften zur Ägyptologie 2, Berlin

Žabkar, L.V. 1975: Apedemak Lion God of Meroe. A Study in Egyptian-Meroitic Syncretism, Warminster 

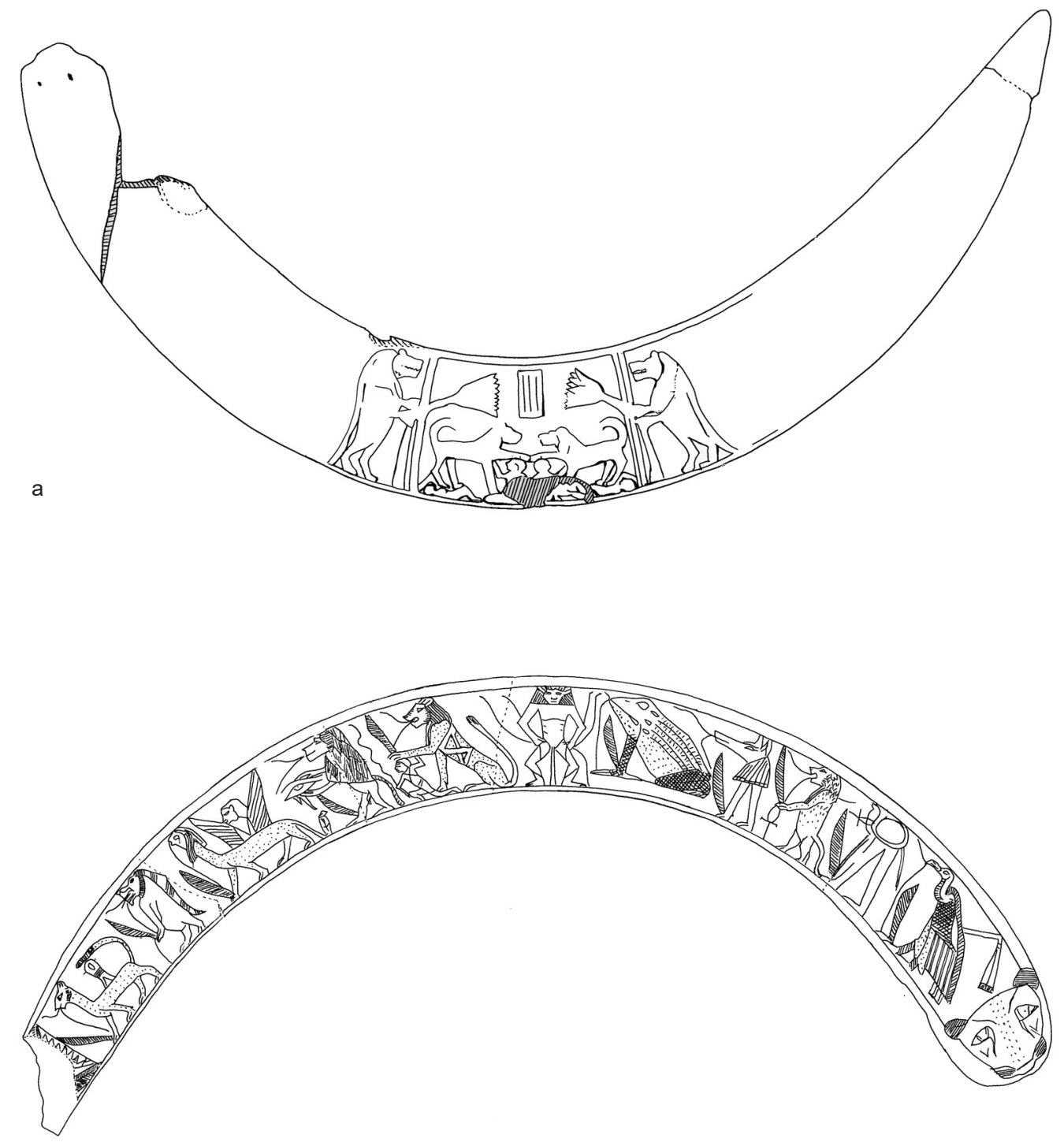

b

1. Zauberstab München ÄS 2952, Vorderseite (a) und Baltimore WAM 71510, Vorderseite (b) (Zeichnung: a. S. Dürr; b. C. Bronkhorst). 


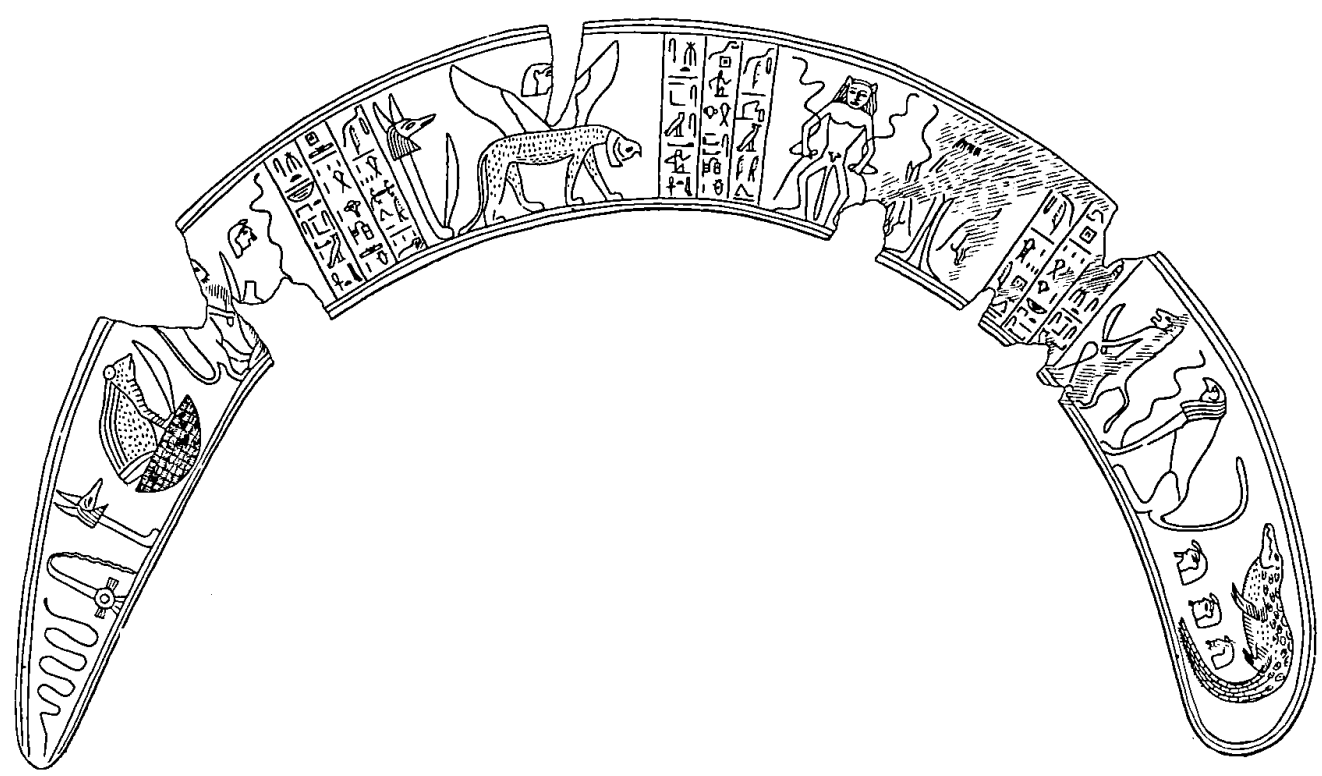

a

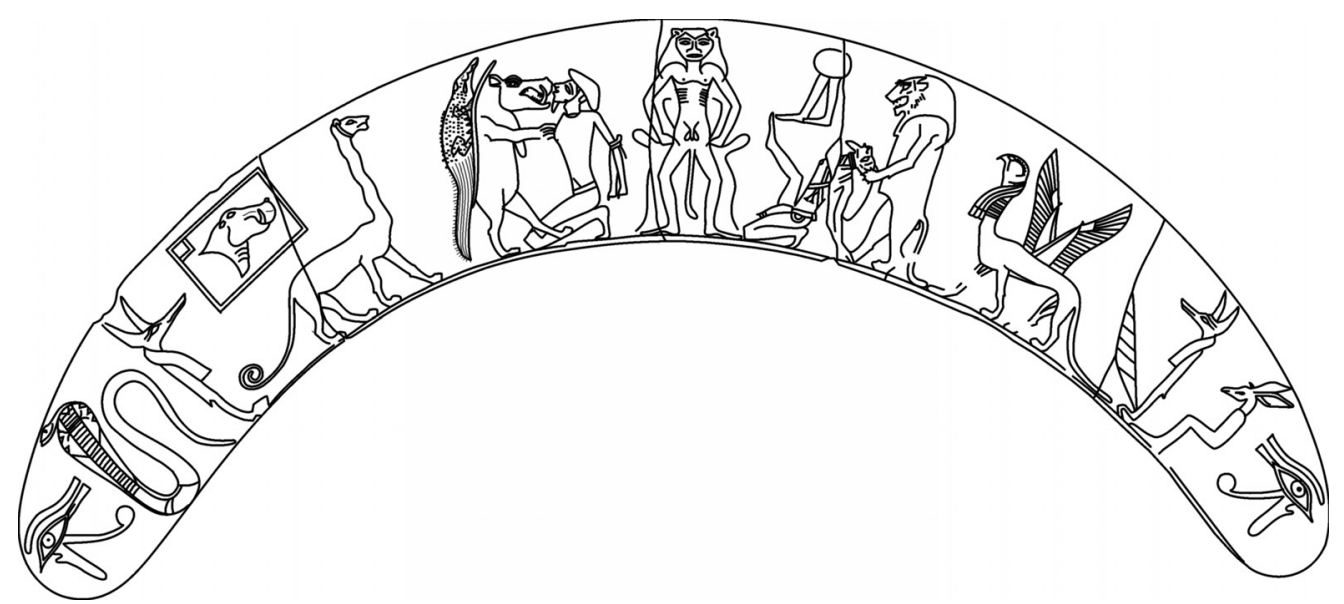

b

2. Zauberstab Berlin ÄM 14207, Rückseite (a) und Louvre E 3614 + MMA 26.7.1288 a-b, Vorderseite (b) (a. nach Legge 1905: 136ff., Taf. IV; b. Zeichnung: C. Preuss-Altenmüller). 


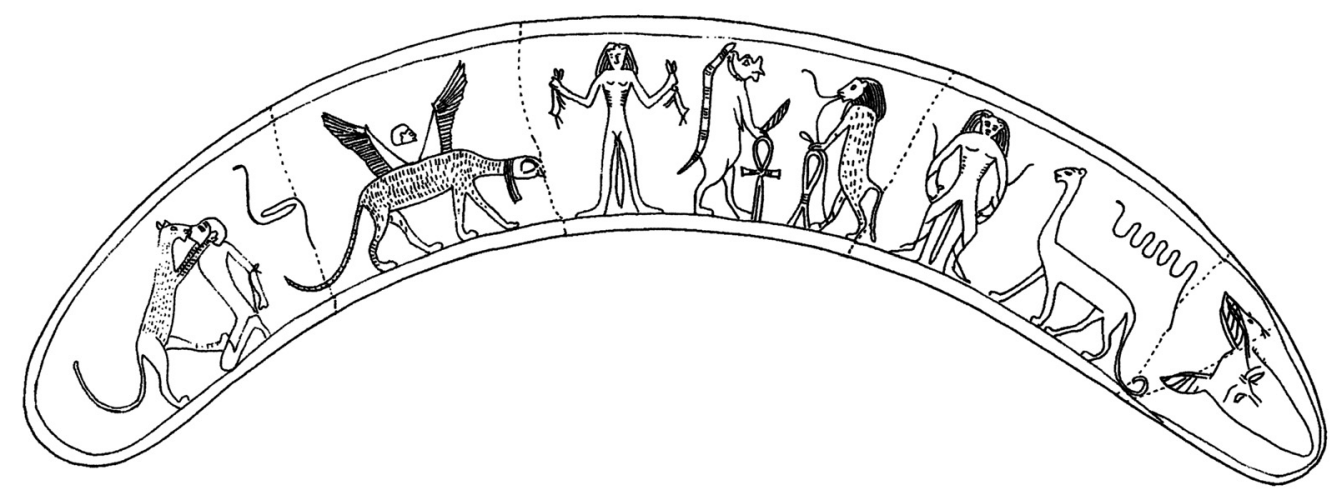

a

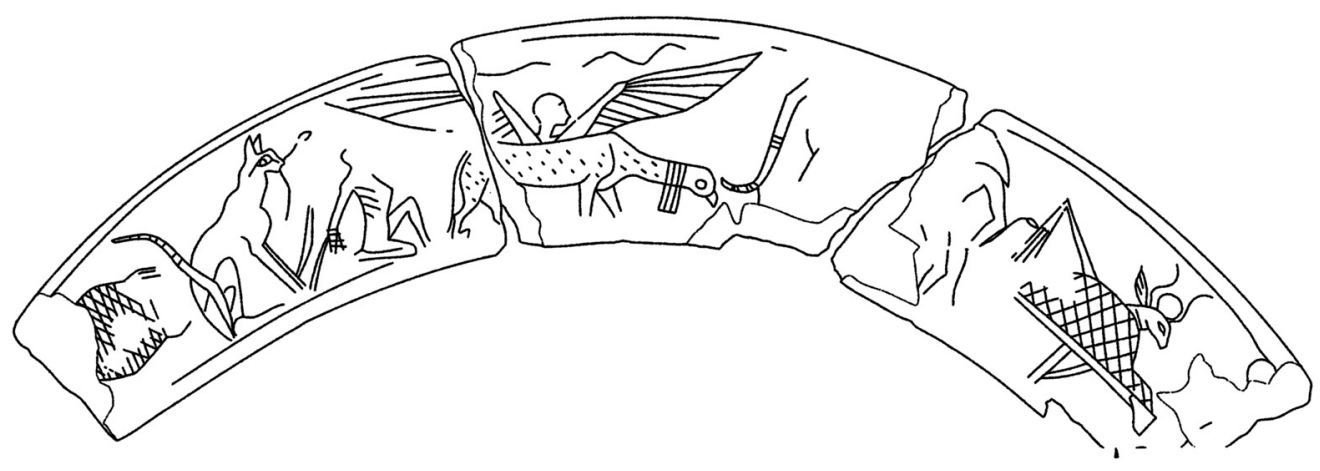

b

3. Zauberstab MMA 15.3.197, Rückseite (a) und Louvre E 20611, Vorderseite (b) (Zeichnung: a. S. Dürr; b. C. Preuss-Altenmüller, nach: Vandier 1969: 43-44, Abb. 3). 


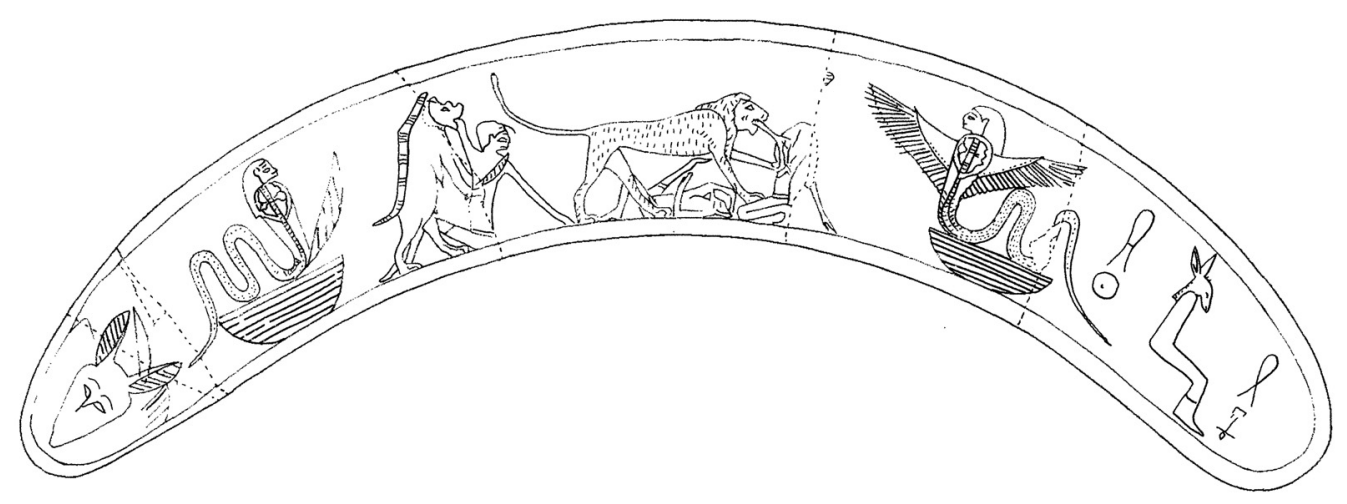

a

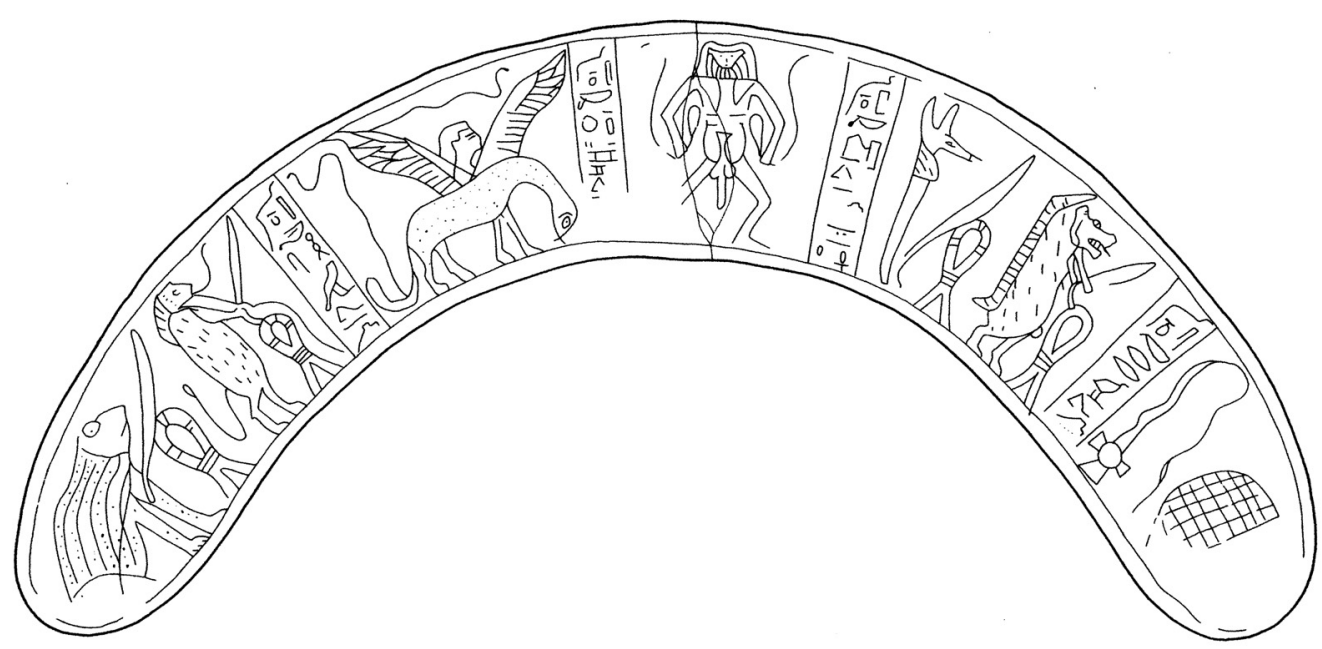

b

4. Zauberstab MMA 15.3.197, Vorderseite (a) und Kairo E. 2007.04.58, Vorderseite (b) (a. Zeichnung: S. Dürr; b. nach Polz et al. 1999: 391, Abb. 18). 


\section{ÉTUDES et TRAVAUX XXX / 2017}

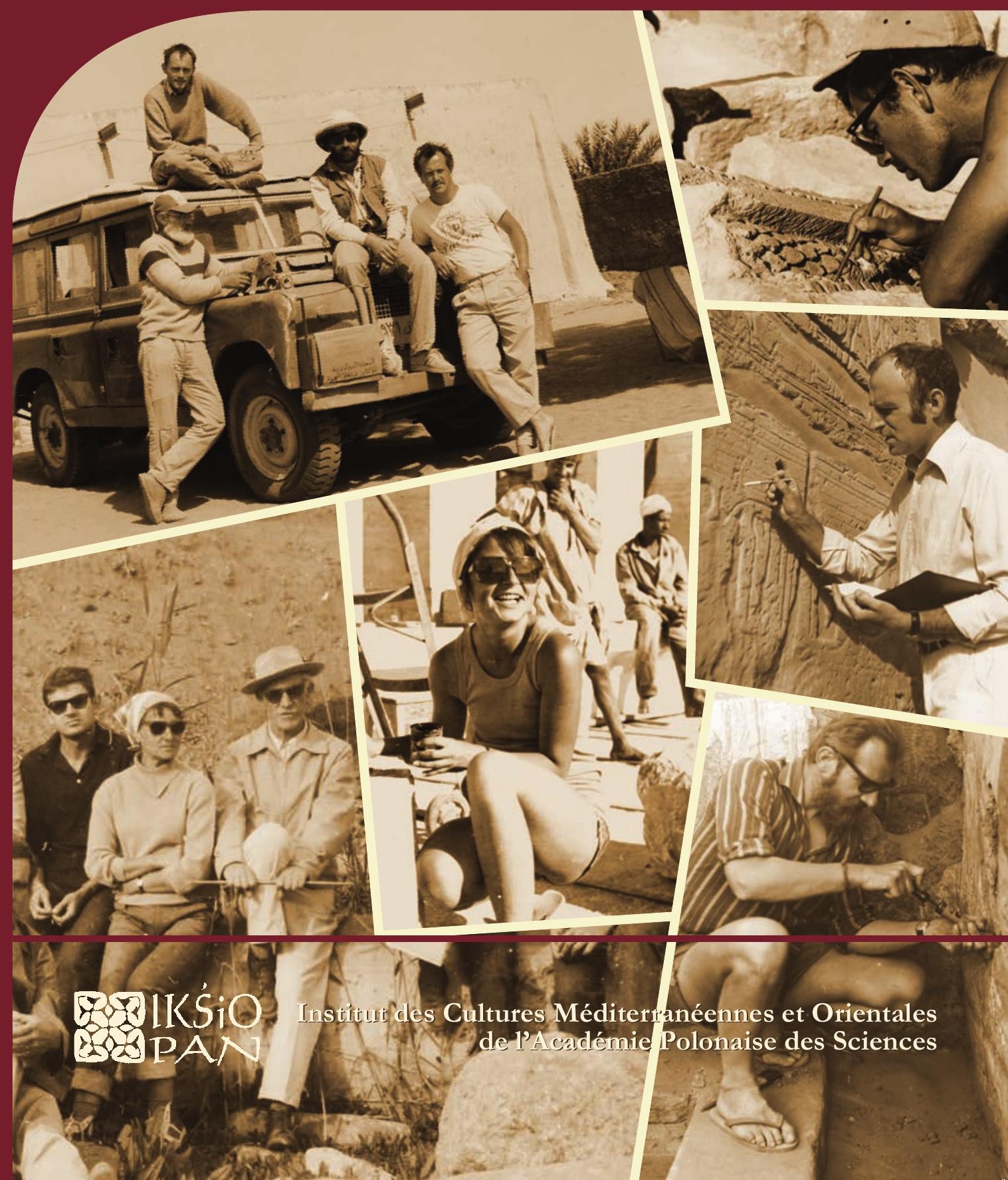




\title{
COMITÉ DE RÉDACTION SCIENTIFIQUE
}

Maciej Makowski - rédacteur en chef

Jadwiga Iwaszczuk - rédacteur et sécretaire de la rédaction

Mariusz Drzewiecki - rédacteur

Maciej G. Witkowski - rédacteur

\section{CONSEIL SCIENTIFIQUE DU JOURNAL}

M. Kobusiewicz (IAE PAS, Warszawa), E. Laskowska-Kusztal (IMOC PAS, Warszawa),

D. Michaelides (University of Cyprus, Nicosia),

J.Ch. Moretti (IRAA-MOM, Université de Lyon 2/CNRS),

D. Raue (Ägyptisches Museum der Universität Leipzig), P. Reynolds (ICREA, Barcelona),

D. Welsby (British Museum, London)

\section{COMITÉ SCIENTIFIQUE DE LECTURE}

J. Holaubek (Institut für Ägyptologie, Wien), S. Ikram (AUC, Cairo),

K. Innemée (Universiteit Leiden), J. McKenzie (Faculty of Oriental Studies, University of Oxford),

N. Strudwick (University of Cambridge), A. Loprieno-Gnirs (Universität Basel),

Ch.E. Loeben (Museen für Kulturgeschichte, Hannover), Y. Tristant (Macquarie University, Sydney),

V.W.J. van Gerven Oei (University of Aberdeen), A. Peignard-Giros (HiSoMA-MOM, Université de Lyon 2/CNRS), J.A. Ostrowski, E. Papuci-Władyka, J. Śliwa (IA JU, Kraków), R. Czerner (WUST, Wrocław), A. Ćwiek (IA AMU, Poznań), M. Wiewióra (IA NCU, Toruń), K. Domżalski

(IAE PAS, Warszawa), K.O. Kuraszkiewicz (DE FOS UW), M. Barwik, P. Bieliński, P. Dyczek, W. Godlewski, D. Ławecka, S. Rzepka, J. Żelazowski, M. Gawlikowski, J. Młynarczyk, A. Niwiński, T. Sarnowski, D. Szeląg, T. Waliszewski (IA UW, Warszawa)

\section{RÉDACTEUR THÉMATIQUE DU VOLUME \\ Barbara Lichocka}

\author{
AIDE RÉDACTION TECHNIQUE \\ Dorota Dobrzyńska, Mariusz Drzewiecki
}

REVUE DES TEXTES ANGLAIS

Jo Harper 
ÉTUDES et TRAVAUX XXX 
INSTYTUT KULTUR ŚRÓDZIEMNOMORSKICH I ORIENTALNYCH POLSKIEJ AKADEMII NAUK

\section{STUDIA i PRACE}

XXX

\section{Ro IKŚSiO \\ ESA PAN}

WARSZAWA

2017 
INSTITUT DES CULTURES MÉDITERRANÉENNES ET ORIENTALES DE L'ACADÉMIE POLONAISE DES SCIENCES

\section{ÉTUDES et TRAVAUX}

XXX

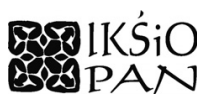

VARSOVIE

2017 
Publication scientifique financée dans le cadre du programme du Ministre de la Science et de l'Éducation Supérieure

« Programme National de Développement de l’Humanistique » pour les années 2016-2021 (projet no 3bH 150099 83)

\title{
HARODOWY PROGRAM ROZWOJU HUMANISTYKI
}

\author{
Copyright (C) \\ Instytut Kultur Śródziemnomorskich i Orientalnych PAN \\ et les Auteurs \\ Warszawa 2017
}

\author{
ISSN 2084-6762 \\ (avant $2011: 0079-3566$ ) \\ e-ISSN 2449-9579 \\ Version première en papier, imprimée en Pologne - 150 copies \\ Version électronique accessible sur \\ http://www.etudesettravaux.iksiopan.pl
}

Édition: Polskie Towarzystwo Historyczne et Wydawnictwo Neriton, Warszawa

Conception générale de couverture : J. Iwaszczuk

Photos de couverture : En haut, à gauche. Vieille Dongola 1991, S. Jakobielski

(debout à gauche), K. Pluskota (debout à droite), B. Żurawski (assis sur le camion)

et P. Wierzbicki (assis sur le camion) (de la collection de B. Żurawski)

En haut, à droite. Palmyre 1964, M. Marciniak au travail (phot. A. Dziewanowski)

Au centre. E. Laskowska-Kusztal au travail (de la collection de E. Laskowska-Kusztal)

En bas, à gauche. Tell Atrib 1962 ; de gauche : T. Biniewski, M. Marciniak, K. Kołodziejczyk,

K. Michałowski, A. Ostrasz, S. Jakobielski et S. Jasiewicz devant eux

(de la collection de IKŚSiO PAN).

En bas, à droite. Vieille Dongola 1976, S. Jakobielski nettoyant le mur (phot. M. Steinborn).

Au centre, à droite, K. Myśliwiec en train des travaux de documentation (de la collection de IKŚiO PAN) 


\section{Table des matières}

BARBARA LICHOCKA

Ergon agathon

Hartwig Altenmüller

$\mathrm{Zu}$ den Feindbildern auf den Zauberstäben des Mittleren Reiches und der Zweiten

Zwischenzeit

Nathalie Beaux

Des $m s w n s w$ de Thoutmosis III à Deir el-Bahari

Briant Bohleke, Nigel Strudwick

A Label for Opening of the Mouth Implements from the Burial of Senneferi (TT99)

and Remarks on the Ritual

Rosa Maria Bonacasa Carra, Nicola Bonacasa

Nuovi dati sugli edifici termali di Sabratha

EDWARD BROVARSKI

A Fragmentary Carrying Chair Scene in Salt Lake City, Utah

Julia Burdajewicz

Wall Painting Decoration from the North-West Church in Hippos-Sussita

of the Decapolis

Mariusz BURDAJEWICZ

From Pagan Temple to Church in Late Antiquity Palestine. A View from

Hippos-Sussita

MAREK ChlodNicki

Early Dynastic Bead Workshops at the Central Kom of Tell el-Farkha.

Patryk ChudziK, Mariusz Caban

Observations on the Architecture of the Tomb of Horhotep in Western Thebes

Krzysztof M. Cialowicz

New Discoveries at Tell el-Farkha and the Beginnings of the Egyptian State.

Amr EL-TiebI

Four Wooden New Kingdom Female Statuettes in the Egyptian Museum, Cairo 


\section{Naguib KanaWATI}

Ritual Marriage Alliances and Consolidation of Power in Middle Egypt during the Middle Kingdom

Adam Łajtar, Jolanta Mlynarczyk

A Faction Acclamation Incised on a Pithos Found Near the North-West Church at Hippos (Sussita)

Adam ŁaJTAR, Grzegorz OchaŁa

Two Private Prayers in Wall Inscriptions in the Faras Cathedral

Adam Łajtar, Anna Poludnikiewicz

Medicinal Vessels from Tell Atrib (Egypt)

JaCeK Michniewicz, Jolanta MlynarczyK

Petrographic Variability of the Fabrics of Wine Jars from Sha'ar-Ha Amakim as a Reflection of Differences in Their Provenance and Chronology

Iwona ModrzewsKa-PianetTI

Les importations d'amphores Dressel 20 en Gaule Cisalpine

Arthur SEgal

Samaria-Sebaste. Portrait of a polis in the Heart of Samaria 409

JOACHIM ŚLIWA

The Motif of a 'Blind Harper' in an Unexpected Place

MONIKA WIĘCH

Searching for the Kitchen in the Early Roman Phase of the 'Hellenistic' House at Nea Paphos (Cyprus)

Abréviations 
THE VOLUME IS PUBLISHED TO CELEBRATE

THE $60^{\text {TH }}$ ANNIVERSARY

OF THE ESTABLISHMENT OF

THE RESEARCH CENTRE FOR MEDITERRANEAN ARCHAEOLOGY POLISH ACADEMY OF SCIENCES

FOUNDED IN 1956

WHOSE MISSION IS CONTINUED BY

THE INSTITUTE OF MEDITERRANEAN AND ORIENTAL CULTURES

OF THE POLISH ACADEMY OF SCIENCES 